\title{
Visuo-Motor Feedback Modulates Neural Activities in the Medulla of the Honeybee, Apis mellifera
}

\author{
Claire Rusch, Diego Alonso San Alberto, and Jeffrey A. Riffell \\ Department of Biology, University of Washington, Seattle, Washington 98195
}

Behavioral and internal-state modulation of sensory processing has been described in several organisms. In insects, visual neurons in the optic lobe are modulated by locomotion, but the degree to which visual-motor feedback modulates these neurons remains unclear. Moreover, it also remains unknown whether self-generated and externally generated visual motion are processed differently. Here, we implemented a virtual reality system that allowed fine-scale control over visual stimulation in relation to animal motion, in combination with multichannel recording of neural activity in the medulla of a female honeybee (Apis mellifera). We found that this activity was modulated by locomotion, although, in most cases, only when the bee had behavioral control over the visual stimulus (i.e., in a closed-loop system). Moreover, closed-loop control modulated a third of the recorded neurons, and the application of octopamine (OA) evoked similar changes in neural responses that were observed in a closed loop. Additionally, in a subset of modulated neurons, fixation on a visual stimulus was preceded by an increase in firing rate. To further explore the relationship between neuromodulation and adaptive control of the visual environment of the bee, we modified motor gain sensitivity while locally injecting an $\mathrm{OA}$ receptor antagonist into the medulla. Whereas female honeybees were tuned to a motor gain of -2 to 2 (between the heading of the bee and its visual feedback), local disruption of the OA pathway in the medulla abolished this tuning, resulting in similar low levels of response across levels of motor gain. Our results show that behavioral control modulates neural activity in the medulla and ultimately impacts behavior.

Key words: electrophysiology; honeybee; sensorimotor; vision; visual learning; visual processing

\section{Significance Statement}

When moving, an animal generates the motion of the visual scene over its retina. We asked whether self-generated and externally generated optic flow are processed differently in the insect medulla. Our results show that closed-loop control of the visual stimulus modulates neural activity as early as the medulla and ultimately impacts behavior. Moreover, blocking octopaminergic modulation further disrupted object-tracking responses. Our results suggest that the medulla is an important site for context-dependent processing of visual information and that placing the animal in a closed-loop environment may be essential to understanding its visual cognition and processing.

Received July 14, 2020; revised Jan. 26, 2021; accepted Jan. 28, 2021

Author contributions: C.R. and J.A.R. designed research; C.R. and D.A.S.A. performed research; C.R. and D.A.S.A. analyzed data; C.R. wrote the paper.

Funding for this research was provided by the Human Frontiers in Science Program (Grant HFSP-RGP0022 to J.A.R.), the National Institutes of Health (Grant 1R21-Al-137947 to J.A.R.), and the Air Force Office of Scientific Research (Grants FA9550-14-1-0398 and FA9550-16-1-0167 to J.A.R.), a Robin Mariko Harris Graduate Fellowship in Insect Studies to C.R., a WRF-Hall Fellowship Award to C.R., and a Lynn Riddiford and James Truman Endowed Fellowship to C.R. We thank the members of the Riffell laboratory, John Tuthill, and Clément Vinauger for support and helpful discussions; Gabriella Wolff for assistance with immunohistochemistry; Brian Smith and Eliot Brenowitz for their help with honeybee rearing; and Nathan Kutz for discussion on data analysis.

C. Rusch's present address: Champalimaud Neuroscience Programme, Champalimaud Center for the Unknown, Lisbon, Portugal.

The authors declare no competing financial interests.

Correspondence should be addressed to Jeffrey A. Riffell at jriffell@uw.edu.

https://doi.org/10.1523/JNEUROSCI.1824-20.2021

Copyright $\odot 2021$ the authors

\section{Introduction}

The response of an animal to a stimulus often depends on the context under which the stimulus is processed. This context may depend on physiological state, or other conditions associated with the sensory environment. For example, the influence of internal state on vision has been extensively studied in the visual cortex of vertebrates, including primates (Nobre and Kastner, 2014) and mice (Khan and Hofer, 2018; Koay et al., 2019), where both body motion and features of the visual environment impact neural responses. In insects, the locomotion state (e.g., walking, running, or flying) also modulates visual processing in multiple brain regions, including the central complex and lobula region of the optic lobe (Paulk et al., 2015; De Bivort and Van Swinderen, 2016; Green et al., 2017). However, the degree to which self-motion modulates upstream visual brain loci remains less well understood (Tuthill et al., 2014). 
The small brains of insects are particularly well suited for studying the links between sensorimotor context, neural processing, and behavior. With these tractable and comparatively simple brains, insects nevertheless exhibit diverse behaviors that are integrally linked to sensorimotor state. For instance, free-flying forager honeybees (Apis mellifera) can learn the concept of sameness and difference (Giurfa et al., 2001), and can discriminate different human faces (Dyer et al., 2005). However, visual learning abilities are dramatically reduced when bees are tethered (Hori et al., 2006, 2007; Sakura et al., 2012) or when head motion is restrained (Niggebrügge et al., 2009; Mota et al., 2011). Recent studies have demonstrated that allowing a bee to walk or move its head while placed in a virtual reality environment increases learning success (Rusch et al., 2017; Buatois et al., 2017). These results suggest that sensorimotor feedback is critical for visual learning in honeybees.

The spatial and temporal stimuli an insect experiences as it navigates will depend on features of its surroundings and the dynamics of its locomotion. In interactions with the environment, the ability to modify behavioral output to generate the expected consequences requires closed-loop control of behavior and is critical for survival. This closed-loop control of behavior has important implications for neural processing of sensory stimuli and the sensitivity of an insect to feedback control. For instance, there is growing evidence that flying a straight path is primarily an operant behavior in the fruit fly Drosophila melanogaster (for review, see Brembs, 2009). Recently, researchers have trained bumblebees (Bombus spp) to adjust their motor actions to perform complex learning tasks, such as successfully pulling strings or rolling balls to obtain a reward (Alem et al., 2016; Loukola et al., 2017). Such studies demonstrate that the insect sensorimotor system continually updates behavior to achieve goal-oriented tasks. Although feedback sensitivity to visual stimuli has been well characterized (Wolf and Heisenberg, 1991; Wolf et al., 1992; Heisenberg et al., 2001; Bahl et al., 2013), the interplay between motor gain and neural processing is less clear (Wolf and Heisenberg, 1986; Fenk et al., 2014; Paulk et al., 2015).

Octopamine (OA), a well known neuromodulator in many insect species, is integrally linked to locomotion state and facilitates the processing of images at increased rates during locomotion (Roeder, 1999; Chiappe et al., 2010; Maimon et al., 2010; Suver et al., 2012; Tuthill et al., 2014). For example, in flies, the release of OA during flight increases gain in lamina and lobula plate neurons (Suver et al., 2012; Tuthill et al., 2014; Strother et al., 2017). However, whether self-generated versus externally generated motion modulates neural activity in the medulla is still unclear, as is the interaction between OA and motor gain in tuning visually evoked responses. In this study, we ask (1) how optic flow impacts neural processing in the honeybee medulla, (2) how behavior is impacted by the relation between honeybee motion and visual input, and (3) whether OA is involved in the observed modulation. Together, our results show that neural responses in the medulla are strongly modulated by sensorimotor feedback, and that $\mathrm{OA}$ is critical for the sensitivity of motor gain at both the behavioral and neural levels.

\section{Materials and Methods}

Honeybees collection and preparation

Sixty-eight forager female honeybees were used in electrophysiological and behavioral experiments between 2018 and 2020 ( 11 bees for the neuronal recording experiments, 57 for the behavioral experiments including 21 for the local-injection pharmacology experiment). Honeybees were either collected from the University of Washington campus (Seattle, WA) or were shipped from Arizona State University (Tempe, $A Z$ ). Based on the behavioral experiments, there were no significant differences between bees from these locations (two-group Mann-Whitney $U$ test; distance walked: Seattle vs Tempe, $U=7,586 ; p=0.437, n=20$ and 16 , respectively). Bees were kept at $25 \pm 1^{\circ} \mathrm{C}$ and under $50 \pm 5 \%$ relative humidity in containers $(11 \times 7.5 \times 8.5 \mathrm{~cm})$, with a $30 \%(\mathrm{w} / \mathrm{w}) \mathrm{su}-$ crose solution (Sigma-Aldrich) available ad libitum. Before experiments, bees were anesthetized on ice and tethered by the thorax and head to a custom 3D-printed holder using UV-activated glue [3:1 mix: Loctite 3104 Light Cure Adhesive; UV Glue GL114 (JewelrySupply.com); and dentist wax (Patterson Dental Supply); Fig. 1A]. The custom holder allowed us to precisely cut a small window in the cuticle (from the ocelli to the antennae and from one eye to the other) to access the brain in the electrophysiological or pharmacological experiments. After tethering, bees were fed with up to $30 \mu \mathrm{l}$ of $50 \%$ (w/w) sucrose solution to check for motivation and allowed to recover for at least $20 \mathrm{~min}$ to $1 \mathrm{~h}$ in a dark, warm, and humid environment (temperature, $25 \pm 1{ }^{\circ} \mathrm{C}$; relative humidity, $55 \pm 5 \%$ ). Approximately $7 \%$ of bees were discarded because of the general lack of locomotion activity after recovering and when placed on the locomotion compensator (forward velocity, $<5 \mathrm{~mm} / \mathrm{s}$ over the experiment).

\section{Experimental setup}

The virtual environment is composed of a locomotion compensator, a screen, and a projector (Fig. 1A). The virtual environment has been described in the study by Rusch et al. (2017). Briefly, the locomotion compensator is an omnidirectional treadmill used to measure the planar trajectories (translation and rotation on the plane) of walking bees. The treadmill consists of a light plastic ball ( $55 \mathrm{~mm}$ W9989 Jumbo Table Tennis Balls, SS Worldwide), a custom-designed 3D-printed support structure (adapted from Moore et al., 2014), an aquarium pump (AP-4 air pump, Danner Manufacturing), and two optic laser sensors (ADNS9800 Laser Motion Sensor, Jack Enterprises), sampled at $50 \mathrm{~Hz}$ using a microcontroller (RedBoard, SparkFun Electronics). The locomotion compensator sits at the center of a cylindrical visual arena (frosted mylar; diameter, $20 \mathrm{~cm}$; height, $30.5 \mathrm{~cm}$ ), subtending $250^{\circ}$ with a $110^{\circ}$ opening in the rear to allow access to the bee and electrode insertion. A video projector (600 lumen K132 WXGA DLP LED Projector, Acer) positioned above the arena projects the visual stimuli downward onto the mylar screen. The visual stimuli are either presented stationary, rotated in closed loop as a function of the calculated bee heading (i.e., the visual scene moves laterally with the rotation of the ball), or rotated in open loop based on predefined inputs.

\section{Visual stimuli}

All experiments were conducted in a dark room. Visual stimuli on the screen were single green or blue bars (width, $5 \mathrm{~cm}$; visual angle, $28^{\circ}$ ) projected on a black background, or vertical gratings of bars $\left(28^{\circ}\right)$. Stimuli were presented in open loop (either static or moving in yaw direct at $100^{\circ} \%$ s) or in closed loop. Closed-loop trials using single bars were subsequently replayed in open loop (replay). The stimuli were similar in brightness (blue stimulus: peak at $451 \mathrm{~nm}, 18 \mathrm{lux}, 0.02 \mathrm{~W} / \mathrm{m}^{2}$; green stimulus: peak at $\left.537 \mathrm{~nm}, 21 \mathrm{lux}, 0.03 \mathrm{~W} / \mathrm{m}^{2}\right)$ and surface area $\left(152.5 \mathrm{~cm}^{2}\right)$. All experiments were conducted under red light [light bulb (Bulbrite), $130 \mathrm{~V}, 10 \mathrm{~W}$, red; covered by a red filter (Roscolux Medium Red Lighting Filter, Rosco)]. The visual angles of both stimuli were above the threshold for discrimination against the background and chromatic perception (Giurfa and Vorobyev, 1997). Spectral characteristics of the visual stimuli were obtained by measuring the relative irradiance of each stimulus (USB2000+ Spectrometer, Ocean Optics; calibration light HL-2000, Oceanview Software). Visual stimuli were displayed on a black background, as this elicited strong fixation and phototactic responses (Rusch et al., 2017; Fig. 1D). In addition to the blue and green stimuli, a closed-loop pattern of multiple human-gray bars was presented between trials.

\section{Behavioral experiments}

Three experimental series were performed in the virtual environment: simultaneous behavioral and electrophysiological recording experiments 
A
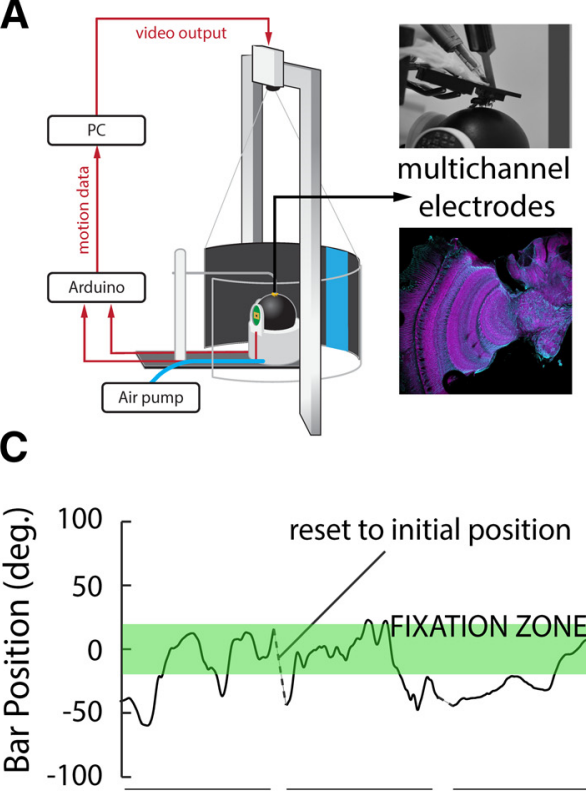

Gain reset to initial position

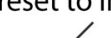

B

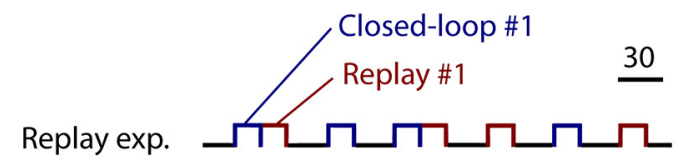

+ bath application octopamine

Gain exp.

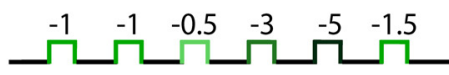

+ local injection epinastine (behavior)

D Single bar-Closed-loop

White backgr. Black backgr.

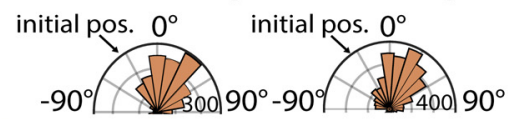

Vertical grating - Closed-loop initial position $0^{\circ}$

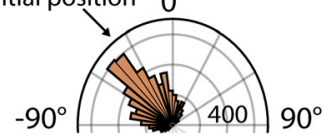

$\mathbf{F}$

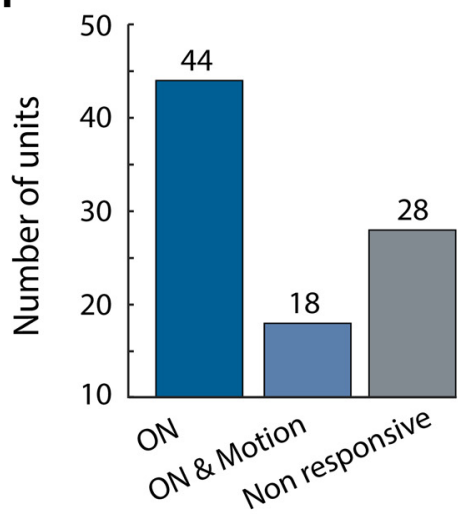

Figure 1. A, Virtual reality environment (adapted from Rusch et al., 2017). A custom-made 3D-printed holder was glued to the head and thorax of the honeybee to prevent head motion, perfuse the brain with saline solution, and ensure walking behavior (insert, top right). The multichannel tetrodes were inserted using a micromanipulator in the medulla of the honeybee, and the reference electrode was lowered into the saline bath near the tetrode. Placement of the electrodes was verified after the experiment (insert, bottom right). $\boldsymbol{B}$, Experimental series: the replay experiment consisted in presenting to the honeybee a single bar in closed loop for $20 \mathrm{~s}$ and subsequently presenting the replay of the visual stimulus motion in open loop. A black screen was presented to the honeybee between trials, and presentations were randomized so that a replay trial did not necessarily follow a closed-loop trial. This experiment series was paired with multichannel electrode recording and bath application of $\mathrm{OA}$ agonist. The second experimental series, the gain experiment, consisted in presenting a single bar to the honeybee at different levels of motor gain. This series of experiments was paired with local injection of epinastine, an $0 \mathrm{~A}$ antagonist, into the medulla. $C$, Single-bar position on the screen during closed-loop trials at different levels of motor gains. The honeybee tends to fixate on the visual stimulus [fixation zone $\left(-20^{\circ}\right.$ to $\left.20^{\circ}\right)$, green rectangle] but fails to do so at the highest level of motor gain $(-3)$. $D$, Position on the screen of the vertical single-bar position over a white or a black background (top) and of the vertical grading (bottom) during closed-loop trials. When presented with a single visual stimulus, honeybees fixated on it, and this behavior was not found when presented with a vertical grating (Watson-Williams test; distribution of angles single bar vs grading, $p<0.001$ ). The fixation to a single visual stimulus did not depend on the background (Watson-Williams test; distribution of angles white vs black background, $p=0.22$ ). $E$, Neuronal activity in responses to visual stimulus (normalized mean firing rate and SEM, top; raster plot, bottom; static stimulus, left; stimulus in motion, right). The insert corresponds to the spike waveform of the corresponding unit. $\boldsymbol{F}$, In total, 90 units were extracted from the neuronal recordings. 44 responded to the presentation of a static visual stimulus, 18 responded to the presentation of a moving visual stimulus, and 28 did not respond to any of the visual stimuli presented. From the 62 responsive units, 7 were excluded from the analysis because of changes in activity over the course of the experiment.

( $n=11$ bees; 90 total units; recording length, $\sim 7200$ s), behavioral experiments alone ( $n=36$ bees; recording length, $1800 \mathrm{~s}$ ), and behavioral experiments coupled with pharmacological manipulation of OA in the bee optic lobe ( $n=21$ bees; recording length, $1200 \mathrm{~s}$ ). The first series was performed to explore the impact of closed-loop control on neural activity in the medulla, whereas the second series examined the impact of gain on the behavioral responses. The last series served to explore further the involvement of $\mathrm{OA}$ in the observed behavioral adaptation to levels of motor gain. All experiments were performed in a dark room and started with a $120 \mathrm{~s}$ acclimation period with a black screen. For all experiments, the forward and angular velocities, the animal heading, and the stimulus position were recorded at $20 \mathrm{~Hz}$. We defined fixation when the stimulus was maintained within $\pm 20^{\circ}$ in front of the honeybee for at least $1 \mathrm{~s}$ while it walked toward the visual stimulus. Visual fixation has been extensively studied in closed-loop paradigm in both walking and flying virtual environments and corresponds to the attempt of an animal to bring a visual stimulus in the center of its field of view (Reiser and Dickinson, 2008; Paulk et al., 2014). For the analysis of the impact of fixation bouts on neural activity, only bees that fixated more than five times throughout the closed-loop presentations were used in the analyses ( $n=9$ bees). Bees fixated, on average, 20 bouts throughout the experiment $(20 \pm 4.14$, mean \pm SEM $)$ and for 2.33 s/bout. 
Open-loop, closed-loop, and replay experiments. In the first series of experiments, we presented the honeybee with either a single green or blue bar that moved across the screen in the yaw direction at $100 \%$ s. We next presented the bar in closed loop for a $20 \mathrm{~s}$ duration, followed by a replay trial where the motion of the closed-loop visual stimulus was repeated to the honeybee in open loop (Fig. 1B). In between presentations, a black screen was presented for $20 \mathrm{~s}$. In all trials, the initial position of the visual stimulus was $-40^{\circ}$, on the left side of the screen, corresponding to the side of the recorded medulla (see below). The number of closed-loop and replay presentations ranged from 16 to 50 per preparation.

Gain experiments. In the second experimental series, we manipulated the gain between the animal motion and the motion of the visual scene to explore further the sensitivity and impact of closed-loop control on visual processing (Fig. 1B). A gain of -1 is the default in our virtual environment and is defined as $1^{\circ}$ change in the animal heading corresponding to a $1^{\circ}$ motion of the visual stimulus in the other direction. Bees were presented a green or blue bar in closed loop at 12 different levels of motor gain, $(-5,-3,-1.5,-1,-0.5,-0.1,+0.1,+0.5,+1,+1.5$, $+3,+5)$. Each presentation lasted for $20 \mathrm{~s}$, and the levels of motor gain were randomly assigned and presented at least four times each. To maintain similar levels of behavioral responses throughout the experiment, between each trial a pattern of gray bars was presented in closed loop for $10 \mathrm{~s}$ at a gain of -1 .

\section{Multichannel recordings}

A hole was cut in the cuticle of the head capsule, as described above, to expose the medulla on both sides of the brain. The brain was superfused continuously with physiological saline solution $(130 \mathrm{~mm} \mathrm{NaCl}, 6 \mathrm{~mm}$ $\mathrm{KCl}, 4 \mathrm{~mm} \mathrm{MgCl}$, $5 \mathrm{~mm} \mathrm{CaCl} 2,160 \mathrm{~mm}$ sucrose, $25 \mathrm{~mm}$ glucose, $10 \mathrm{~mm}$ HEPES, pH 6.7) at room temperature. The multichannel electrode was positioned in the left medulla under visual control using a microscope and a micromanipulator. Recordings were made with a 16-channel silicon multielectrode $(2 \times 2,3 \mathrm{~mm}$, catalog \#150-150-121; and $4 \times 4,3$ $\mathrm{mm}$, catalog \#50-125-177, NeuroNexus). Electrophysiological signals were amplified $10,000 \times$, filtered (low-pass filter, $6 \mathrm{kHz}$; high-pass filter, $300 \mathrm{~Hz}$ ), recorded, and digitized using acquisition software (Tucker-Davis Technologies). Spike data after thresholding were subsequently manually clustered using Offline Sorter (Plexon). Only well clustered units that separated in a three-dimensional space (PC1-3) after statistical verification (multivariate ANOVA, $p<0.05$ ) were used in the analysis. Unit responses were initially examined in Neuroexplorer (NEXTechnologies) before being analyzed through custom-written and open-source code in MATLAB and the Statistics Toolbox (release 2019a, MathWorks). To characterize whether a unit was responsive to the static visual stimulation, we calculated the firing rate during stimulation and compared it with the firing rate immediately before the stimulus onset for the same duration using a Wilcoxon signed-rank test for paired samples $(\alpha=0.05)$. For the moving visual stimulation, we used the same method but compared the firing rate at the onset of the visual stimulation and during the motion of the visual stimulus. For both the static and the moving visual stimulation, units that spiked $<1$ spike/s were excluded from the analysis. During closed-loop and replay experiments, firing rate responses were estimated using bins of $50 \mathrm{~ms}$ and were used to classify units based on their responses to visual stimulation. In total, 90 units from 11 individuals were recorded simultaneously with behavior (mean, 8 units per preparation). From those 90 units, 44 responded to the presentation of the blue or green bars, and 18 responded significantly to visual motion (Fig. $1 E, F$ ). Only 55 units were used to explore the impact of the closed-loop control as their spontaneous activity, and visual-evoked responses were stable over time (Fig. 2C).

\section{Pharmacological experiments}

The OA antagonist (epinastine hydrochloride, Sigma-Aldrich) and agonist (OA hydrochloride, Sigma-Aldrich) were dissolved in saline at concentrations of $4.10^{-3}$ and $10^{-4} \mathrm{M}$, respectively. Epinastine is a highly specific octopamine receptor antagonist in honeybees, with a much lower binding affinity ( $10^{4}$ times) for other bioamine receptors (Roeder et al., 1998). Our epinastine concentrations were similar to those of previous studies in honeybees (Rein et al., 2013; Tedjakumala et al., 2014). Drugs or saline alone (control) were injected either into the brain as a bath application $(\mathrm{OA}, 10 \times 20 \mathrm{nl})$ or directly inside each medulla (epinastine, $2 \times 2 \mathrm{nl}$ ). For the local injection, volumes injected were calibrated before and after injection using a Malassez cell, and injections were performed $15 \mathrm{~min}$ before the experiment started.

\section{Immunohistochemistry}

Microelectrode insertion. To image the insertion of the microelectrodes into the medulla, the tips of the silicon electrodes were coated with a solution of $2 \%$ Texas Red (Thermo Fisher Scientific) mixed in saline. After the experiment, heads were removed into cold $\left(4^{\circ} \mathrm{C}\right)$ fixative containing 4\% paraformaldehyde (PFA) in PBS, pH 7.4 (Sigma-Aldrich) and left overnight at $4^{\circ} \mathrm{C}$. The following day, the heads were washed two times over $20 \mathrm{~min}$ in PBS, and the brains were dissected and then embedded in agarose. The resulting embedded tissue was cut into $60 \mu \mathrm{m}$ serial sections using a vibratome. Sections were washed in PBS containing $0.5 \%$ PBS-Triton X-100 (TX; Sigma-Aldrich) two times over $20 \mathrm{~min}$. Then $50 \mu$ l of normal serum was added to each well containing $1 \mathrm{ml}$ of PBS-TX. After $1 \mathrm{~h}$, the primary antibody was added to each well, and the well plate was left on a shaker overnight at room temperature. The next day, sections were washed six times over $20 \mathrm{~min}$, and $2.5 \mu \mathrm{l}$ of Cys 3 donkey anti-mouse IgG and Alexa Fluor 488 (Thermo Fisher Scientific) was added to $1 \mathrm{ml}$ of PBS-TX in each well, and the well plate was again left on the shaker overnight. Tissue sections were then washed in PBS six times over $3 \mathrm{~h}$ and embedded on glass slides in Vectashield (Vector Laboratories). After at least $24 \mathrm{~h}$ at $4^{\circ} \mathrm{C}$, the brain tissue was imaged using a Leica SP5 laser-scanning confocal microscope, and the resulting image stacks were processed using ImageJ (National Institutes of Health).

Characterization of pharmacological microinjections. To allow us to visualize both the insertion site of capillaries and the spread of the solution after microinjection, we mixed the saline or OA antagonist solution with 2\% Texas Red (Thermo Fisher Scientific). After the experiment, the head of the honeybee was removed and placed in $4 \%$ PFA and left overnight at $4^{\circ} \mathrm{C}$. The following day, the heads were washed six times in PBS for $20 \mathrm{~min}$ each before the brains were removed from the head capsule. Brains were dehydrated in a series of increasing ethanol solutions followed by clearing in methyl salicylate (1:1 with $100 \%$ ethanol, then $100 \%$ methyl salicylate). After at least $24 \mathrm{~h}$ at $4^{\circ} \mathrm{C}$, the brain tissue was imaged using a Leica SP5 laser-scanning confocal microscope. The images were processed using ImageJ (National Institutes of Health).

\section{Data analysis}

Normalized firing rate. To compare firing rate between closed-loop and replay trials, we calculated a normalized difference:

$$
\Delta \text { Firing Rate }=\frac{\text { Mean Firing Rate } \text { Closed-loop }- \text { Mean Firing Rate } \text { Replay }_{\text {Mean Firing Rate }}}{\text { Mlosed-loop }},
$$

where mean firing rate responses for closed-loop or replay stimulus presentations were calculated over a $16 \mathrm{~s}$ window $2 \mathrm{~s}$ after the onset of the stimulus and averaged over 16-50 trials for each unit. To statistically analyze closed-loop and replay responses, for each unit we assessed the difference in $\Delta$ Firing Rate (hereafter: $\Delta \mathrm{FR}$ ) by comparing the closed-loop and replay datasets to a null distribution of 100 bootstrapped pairwise differences randomly drawn from the combined closed-loop and replay visual stimulus datasets.

Generalized linear model. For each responsive unit, we applied the following generalized linear regression model assuming a Poisson distribution, as follows:

$$
\begin{gathered}
\log (\text { Spike count })=1+\text { Stimulus Position }+ \text { Forward Velocity } \\
+ \text { Heading }+ \text { Behavioral Control },
\end{gathered}
$$

where the Stimulus Position variable corresponded to the position of the bar on the screen, the Forward Velocity and Heading corresponded to 
A

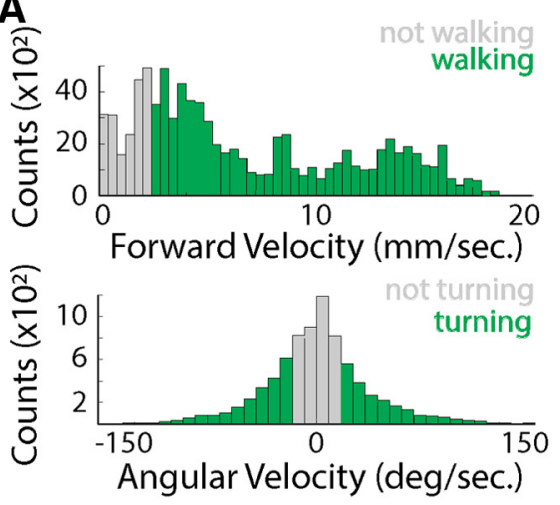

D
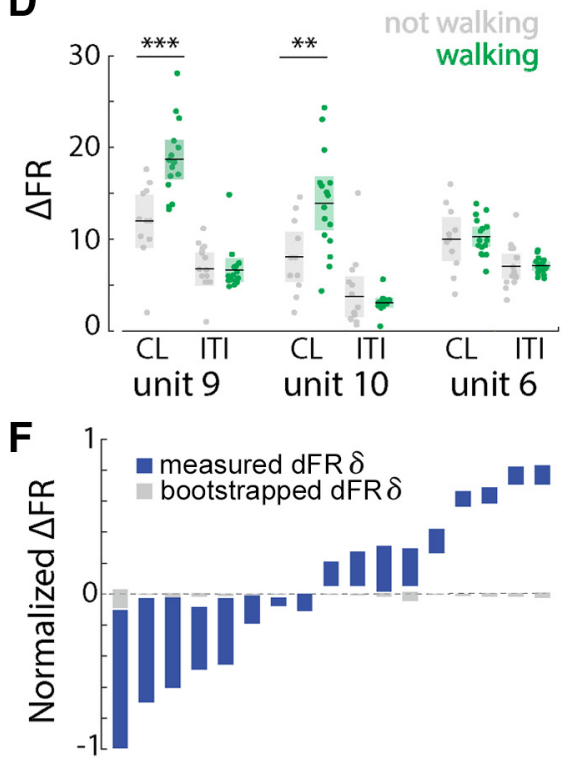

Significant units (sorted)
B
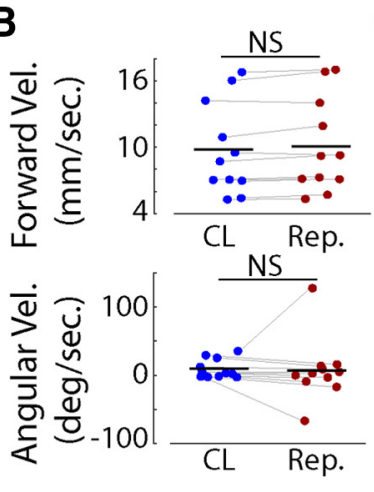

C
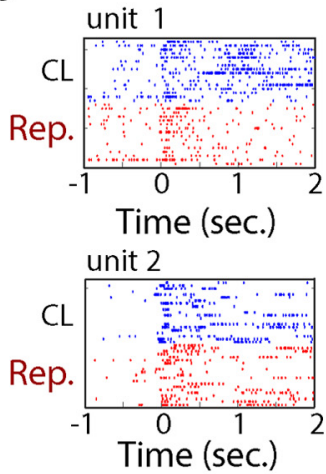

E
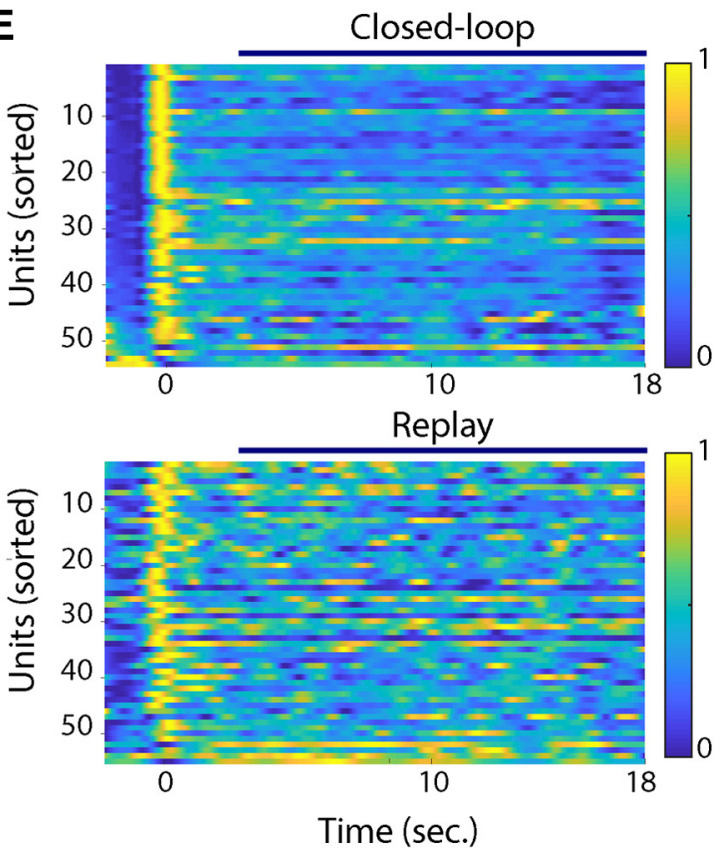

Figure 2. A, Top, Forward velocity (Forward Vel.) distribution for one honeybee. The data were divided in not walking bouts (forward velocity $<2.5 \mathrm{~mm} / \mathrm{s}$, gray bars) and walking bouts (forward velocity $>2.5 \mathrm{~mm} / \mathrm{s}$, green bars). Bottom, Distribution of angular velocity (Angular Vel.) for one honeybee. The data were divided into turning bouts (angular velocity $>20^{\circ} / \mathrm{s}$ or less than $-20 \%$, green bars) and not turning bouts (angular velocity between $-20^{\circ}$ and $20 \%$, gray bars). $\boldsymbol{B}$, Top, Forward velocity averaged across closed-loop or replay trials for each bee (Wilcoxon rank-sum test: $z=-1.761, p=0.07$; each dot $=1$ bee). Bottom, Angular velocity averaged across closed-loop or replay trials for each bee (Wilcoxon rank-sum test: $z=0.56$, $p=0.57$; each dot $=1$ bee) $\boldsymbol{C}$, Neural responses (raster plots) of two units during closed-loop (blue) and replay (red) trials. Unit 1 shows a decreased rate of spiking during the replay trials. $\boldsymbol{D}$, For three different units, the mean firing rate (in spikes per second) during walking (green) and nonwalking (gray) bouts, averaged for each trial of closed-loop (CL) and ITIs where no visual stimulus (black screen) was presented to the honeybee. Shaded rectangles represent the $95 \%$ confidence interval. Units 9 and 10 showed a significant increase in firing rate during walking bouts and in the presence of visual feedback (Kruskal-Wallis test: $* * p<0.01, * * * p<0.001$ ). $\boldsymbol{E}$, Averaged response rates for all 55 recorded units for closed-loop trials (top) and the replay trials (bottom). Units were ordered by their response in closed loop at time 0 s. $\boldsymbol{F}$, Normalized difference in firing rate between closed-loop and replay trials for the units that showed a significant difference from 0 (Wilcoxon rank-sum test, $p<0.05$ ) and from a bootstrap analysis (Wilcoxon rank-sum test, $p<0.05$ ). The colored bars show the $95 \%$ confidence interval for the $\Delta$ FR, and the gray bars represent the $95 \%$ confidence interval for the bootstrapped data. NS, not significant.

the instantaneous forward velocity and heading of the animal at the same instant, and the Behavioral Control (BC) variable corresponded to the level of motor gain during the trial, either open loop (replay) or closed loop. All variables were recorded at $20 \mathrm{~Hz}$, and the model was trained on $80 \%$ of the dataset and the $20 \%$ left were used to predict the firing rate using the trained model. The random sampling of the dataset for training and testing was balanced across behavioral control labels (closed-loop and replay trials). For each unit, the model was run 100 times, and the mean of the $p$ values against the null model and of predictors was extracted to assess whether the model was different from a null model and whether the behavioral control was a significant predictor of spiking activity. The resulting Akaike Information Criterion (AIC) values were used to evaluate the different variables (with and without Behavioral Control) on the model results.

Singular value decomposition. To reduce the dimensions in our dataset, we applied singular value decomposition (SVD), where the mean-centered matrix $A$ of the normalized firing rate was as follows:

$$
A=U \Sigma V^{*}
$$

The $U$ and $V$ matrices are orthogonal and unitary, and their columns correspond to the eigenvectors of $A A^{T}$ and $A^{T} A$. The $U$ matrix corresponds to the modes of the decomposition, and the weight associated with each mode is given by their corresponding singular values (diagonal of the matrix $\Sigma$ ). The SVD was applied either to a population of neurons or a single unit. When applied to a population of neurons, the meancentered matrix $A$ corresponded to the normalized mean across trials for each unit. When applied to a single unit, the mean-centered matrix $A$ corresponded to the firing rate under different conditions (e.g., fixation bouts and randomly selected bouts). We then compared the energy contained in each mode across the different conditions. 
k-nearest neighbor classification. To build a model that would classify and predict behavioral control based on the firing rate of the population, we separated the matrix into two subsets, one containing $80 \%$ of the data for training our model, and the other $20 \%$ for testing. We then applied the SVD to the training matrix to extract the most essential features. The testing matrix was projected on the SVD space obtained with the training matrix to obtain the $V_{\text {test }}$ matrix:

$$
V_{\text {test }}=U^{\prime} A_{\text {test }} \text {. }
$$

A k-nearest neighbor classifier algorithm using the three closest neighbors was trained with the matrix $V$ and tested with the matrix $V_{\text {test. }}$ The training-testing steps were repeated 100 times to assess the mean accuracy in both training and testing.

Gain experiment behavioral data analysis. The data collected (stimulus position, animal heading, and velocity) in the gain experiment were analyzed, and all statistical tests were computed using $\mathrm{R}$ software ( $\mathrm{R}$ Core Team, 2017) and the circular package. A random walk model was developed to ensure that the difference in the duration of fixation bouts observed was not only because of the difference in the animal velocity. In the model, a fictive animal can take a step in one of three different directions (left, right, forward) with the same probability at every timestamp, and the "amplitude" of the step was randomly drawn from the angular velocity distribution of the epinastine- and saline-injected bees.

\section{Results}

\section{Locomotion state and turning behavior impact neuronal activity in the medulla}

To explore the impact of locomotion on neuronal responses in the optic lobe, we placed tethered, walking bees in a virtual reality arena while performing multichannel recording in the bee medulla (Fig. 1A). Visual stimuli included static and moving vertical gratings or single bars (size, $28^{\circ}$; velocity, $100^{\circ} / \mathrm{s}$ ) that were presented in closed-loop trials and subsequently replayed in open-loop trials (Fig. 1B). Bees exhibited robust fixation responses to all visual stimuli, and during closedloop trials they maintained the visual stimulus in the frontal region of the eyes (Fig. $1 C, D$ ). Based on the single-unit responses to these visual stimuli, $\sim 69 \%$ of neural units were sensitive to the onset of a colored visual stimulus, or to the onset and the motion of a colored visual stimulus (Fig. 1E,F).

While walking speed is known to modulate neural activity in insect optic lobes (Chiappe et al., 2010; Paulk et al., 2015), it is unclear how closed-loop control impacts early brain loci such as the medulla. We examined bee walking and turning behaviors with respect to the position of a visual stimulus, and classified the behavior into active and nonactive bouts based on our observations and recordings. We considered the honeybee to be walking if its forward velocity was at least $2.5 \mathrm{~mm} / \mathrm{s}$. (Fig. $2 A$ ), or turning when its absolute angular velocity was $>20 \%$ s (Fig. $2 A$ ). During closed-loop trials, bees walked $66.25 \pm 8.27 \%$ of the time (mean $\pm \mathrm{SE}$ ), and $66.56 \pm 8.49 \%$ during replay trials. The average forward velocity of walking bouts was $9.48 \pm 1.55 \mathrm{~mm} / \mathrm{s}$ (mean \pm SEM) during closed-loop trials, and $1.14 \pm 0.09 \mathrm{~mm} / \mathrm{s}$ during bouts defined as nonwalking.

We found no difference in walking or turning velocities between closed-loop and replay trials, across all bees and all trials (Fig. 2B; Wilcoxon rank-sum test for closed-loop vs replay trials; forward velocity: $z=-1.761, p=0.07$; angular velocity: $z=0.56, p=0.57)$. However, the locomotion state did impact single-unit responses (Fig. 2D). A significantly greater number of units was modulated during closed-loop turning responses (19 units, 35\% of all units), compared with only 4 units modulated by angular velocity in replay trials (two-way ANOVA, firing rate $\sim$ angular velocity $*$ behavioral control, $p_{\text {angular velocity }}<0.01 ; p_{\text {behavioral control }}<0.01$ ). Similar results occurred when comparing neural responses during closed-loop versus nonvisual stimulus [black screen; intertrial interval (ITI)] trials (two-way ANOVA, firing rate $\sim$ angular velocity $*$ behavioral control, $p_{\text {angular velocity }}<$ $\left.0.01 ; p_{\text {behavioral control }}<0.01\right)$. Walking activity also impacted neural responses. During closed-loop trials, firing rate responses were significantly altered for 8 units $(15 \%$ of units), compared with only 2 units modulated in replay trials or during presentation of the black screen during ITI (twoway ANOVA, firing rate $\sim$ forward velocity $*$ visual stimulus condition, $p_{\text {forward velocity }}<0.01, p_{\text {visual stimuulus condition }}<$ 0.01 ; Fig. 2D).

\section{Characterizing and modeling the impact of behavioral control on single-unit and ensemble responses}

To further characterize the effects of behavioral control on single-unit and ensemble responses, we first compared firing rate responses between closed-loop and replay trials in $50 \mathrm{~ms}$ bins during the interval from 2 to $18 \mathrm{~s}$ (Fig. 2C,E). The binning and time interval were chosen to capture the dynamics of closed-loop control, while excluding any $\mathrm{ON}$ response to the onset of the visual stimulus. In 17 units, firing rate responses differed significantly between the two conditions (Fig. $2 F$; Wilcoxon rank-sum test, $\Delta \mathrm{FR}$ vs $0, p<0.05)$, and this difference could not be explained by the variability in firing rate activity, independent of behavioral control ( $\Delta \mathrm{FR}$ vs bootstrapped $\Delta \mathrm{FR}, p<0.05)$.

To further explore the impact of behavioral control on dynamic responses of medulla neurons, we fitted a generalized linear model to the responses of each unit for all trials (16-50 trials/condition) and compared the model with and without the behavioral control variable (defined as a two-level categorical variable: closed-loop or replay; Fig. $3 A$ ). For 30 units, BC was not a significant predictor of spiking activity (Fig. $3 B$ ). For the remaining 25 units, behavior control was a significant predictor, and the model differed significantly from a null model fit to the same data $(p<0.05)$. For each of those 25 units, we compared the model AICs with or without the control variable. We found that for 14 units, including the behavioral control variable in the model significantly lowered the AIC values (one-tailed Wilcoxon rank-sum test, $\mathrm{AIC}_{\text {withBC }}$ vs $\mathrm{AIC}_{\text {withoutBC }} z<-0.10, p<0.05$; Fig. $3 D$ ) and increased the $R^{2}$ values (one-tailed Wilcoxon rank-

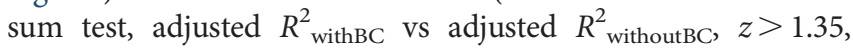
$p<0.05$, Fig. $3 D$ ). The predicted firing rate based on the models for those 14 units captured the observed single-unit responses, and predictions were improved by the inclusion of the behavioral control variable in the model (Fig. $3 D$ ). In contrast, for the other 11 units, including the behavioral control variable in the model did not improve predicted firing rates (Fig. 3C). For these 11 units, it is likely that $\mathrm{BC}$ is not a modulator of the firing rate in response to the visual stimulus and its motion.

We next used dimension reduction techniques (i.e., SVD) to explore the impact of behavioral control at the neural population level. The SVD was applied to a matrix containing the normalized mean firing rate under the following three conditions: closed-loop trials, replay trials, and trials with no visual stimulus presented (Fig. 3E). At the onset of visual stimulus, firing rate responses were both higher and less variable in the closed-loop condition compared with the replay stimulus, which exhibited greater firing rate response variability in the preceding $16 \mathrm{~s}$ window. To exclude ON responses, the SVD was applied to the interval from 2 to $18 \mathrm{~s}$ in the stimulus trials (Fig. $3 E$, blue dots). 
A
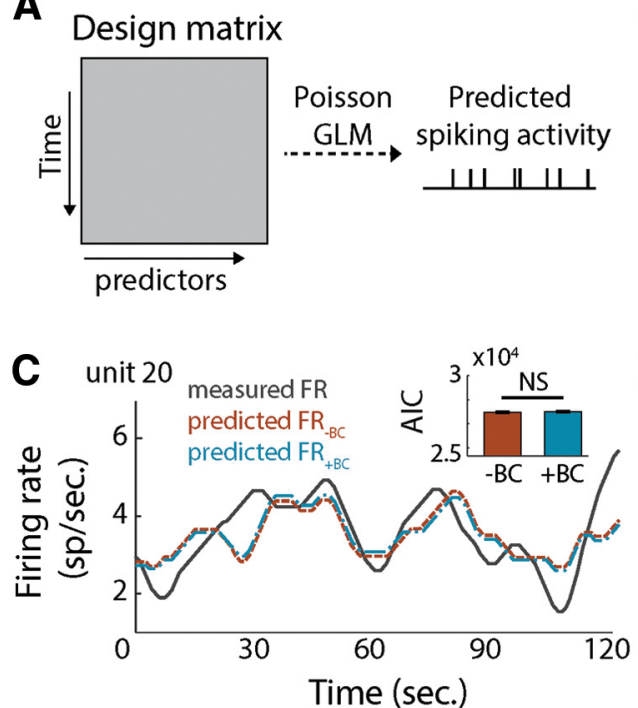

E

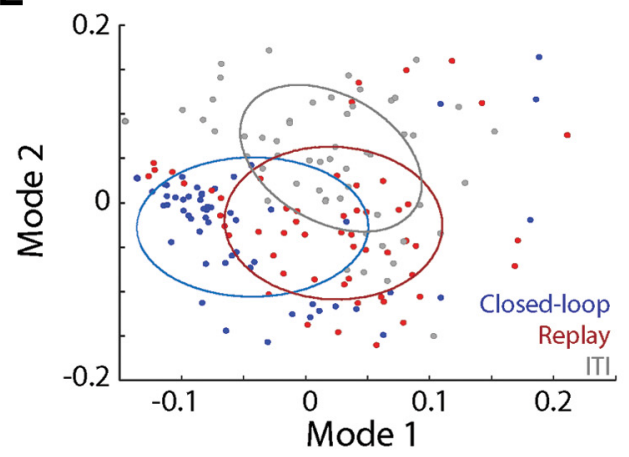

G

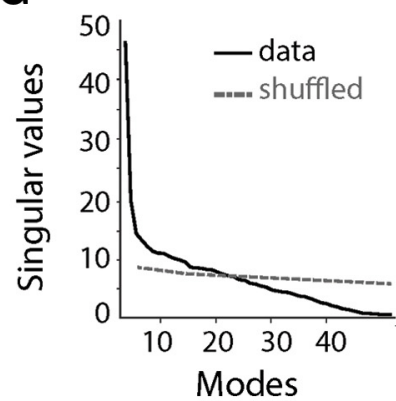

H
B unit 19-non predictive
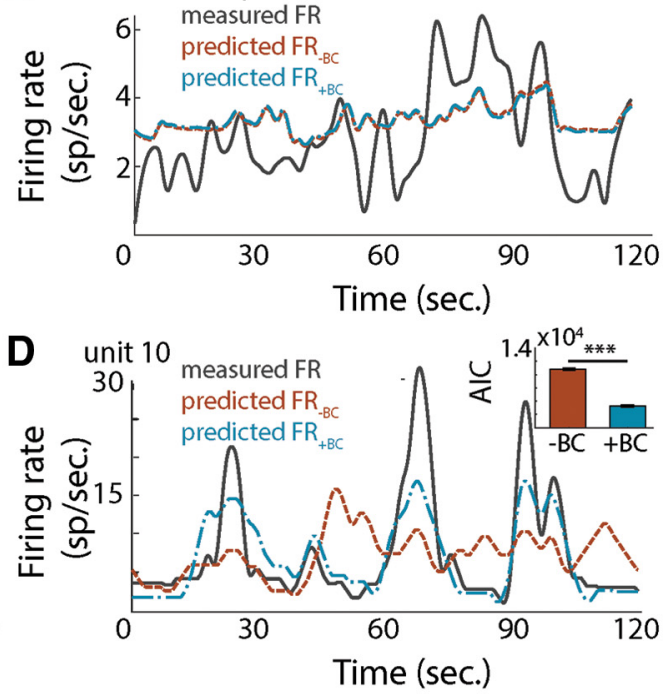

$\mathbf{F}$

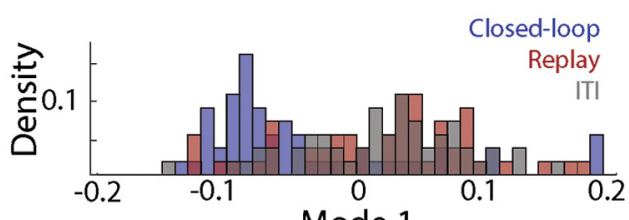

Mode 1
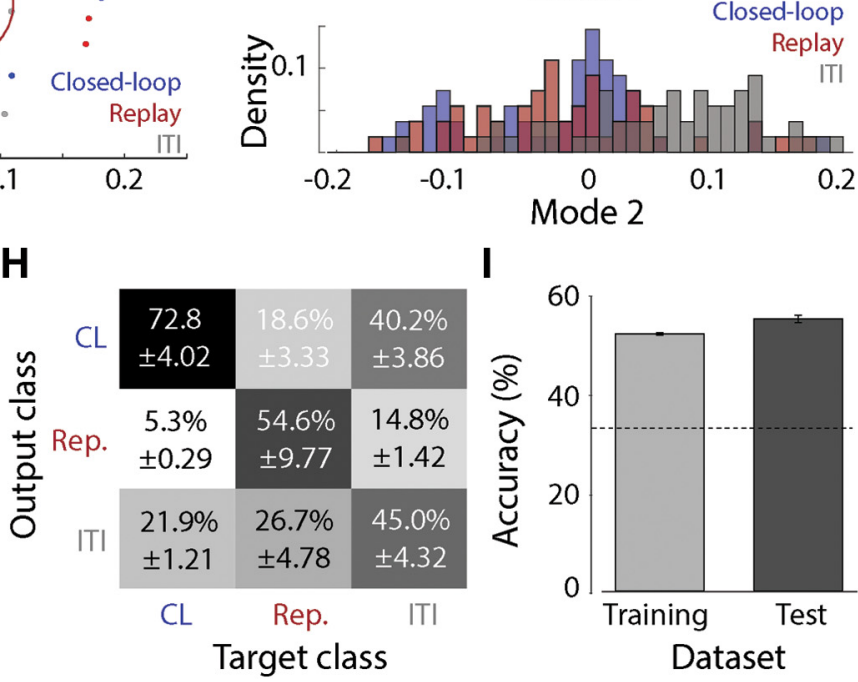

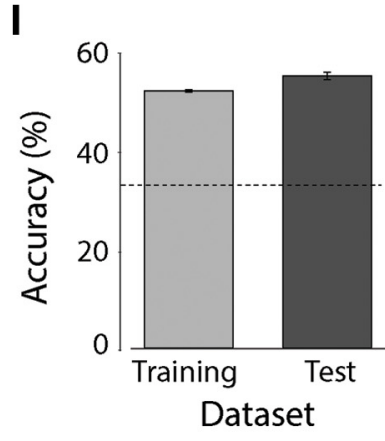

Figure 3. $A$, Generalized linear model. The design matrix contains the predictor variable: stimulus position, forward velocity, heading, spike history, and $B C$. The response variable is the spike count. The model assumed a Poisson distribution of the response variable. $\boldsymbol{B}$, Measured firing rate (gray line) and predicted firing rate without the $B C$ variable (orange line) or with the $B C$ variable (blue) for one unit where the position of the stimulus and the $B C$ variables were not significant predictors. The time course corresponds to a concatenation of the $20 \mathrm{~s}$ bout of different trials. $C$, Measured firing rate (gray line) and predicted firing rate without the $B C$ variable (orange line) or with the $B C$ variable (blue) for one unit where both variables were significant predictors. The time course corresponds to a concatenation of the $20 \mathrm{~s}$ bout of different trials. The insert represents the $A I C$ of the model with and without the $B C$ variable. Inclusion of the $B C$ variable did not significantly impact the $A I C$ of the model (Wilcoxon rank-sum test, $p=0.56$ ). $D$, Measured firing rate (gray line) and predicted firing rate without the $B C$ variable (orange line) or with the $B C$ variable (blue) for one unit where both variables were significant predictors. The time course corresponds to a concatenation of the $20 \mathrm{~s}$ bout of different trials. The insert represents the $A I C$ of the model with and without the $B C$ variable. Inclusion of the $B C$ variable decreased the $A I C$ of the model (one-tailed Wilcoxon rank-sum test, $A I C_{\text {withBC }}$ vs $A I C_{\text {withoutBC }}$ $p<0.001$ ). $\boldsymbol{E}$, Distribution of the closed-loop (blue) and replay (red) trials, and intertrial intervals (gray) in the first mode. The ellipses correspond to the $50 \%$ confidence interval. $\boldsymbol{F}$, Distribution of the closed-loop (blue) and replay (red) trials, and random intertrial intervals (e.g., without presentation of visual stimulus, gray) in the two first modes after singular value decomposition. $\boldsymbol{G}$, Singular values along modes for the firing rates and a shuffled firing rate responses. The first 20 modes of the data contain more information than the ones of the shuffled data. $\boldsymbol{H}$, Confusion matrix of the classification algorithm for the condition closed loop (CL), replay (Rep.) and ITls. $\boldsymbol{I}$, Percentage of accuracy in training and testing for our k-nearest neighbor classification algorithm. The dashed line corresponds to chance level $(0.33)$. $* * * p<0.001$. NS, not significant.

The ensemble responses between the closed-loop and replay treatments were intermixed, but still significantly different from one another in the multivariate space (Fig. 3F). In the first mode of the SVD, closed-loop trials were separate from the replay trials and the unstimulated (i.e., ITI) condition, whereas in the second mode, closed-loop and replay trials were separate from responses to unstimulated condition (Fig. 3F). We next trained a k-nearest neighbors classification algorithm to sort the data in closed-loop, replay, and no visual stimulus trials using the first 20 modes, since these singular values were higher than for a noise model based on a shuffled dataset (Fig. $3 G$ ). Interestingly, the algorithm performed better at correctly assigning the closed-loop 

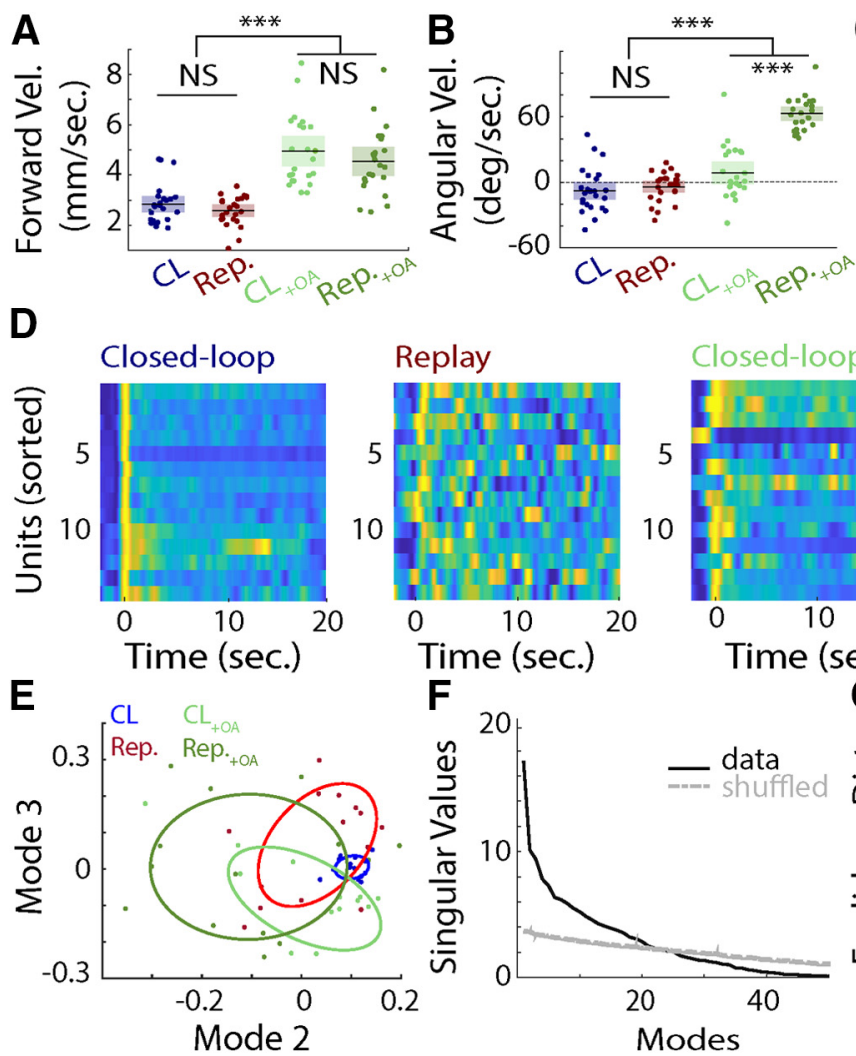

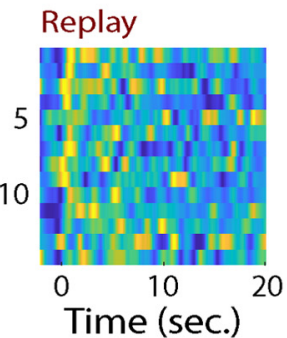

$\mathbf{F}$

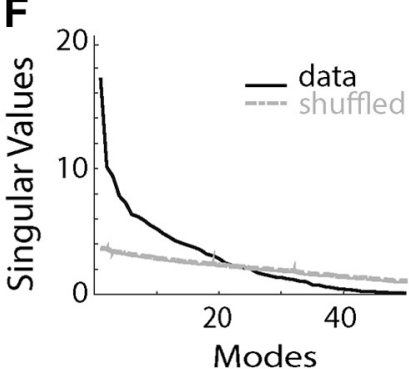

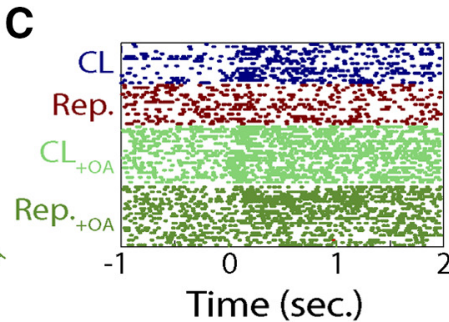

Time (sec.)
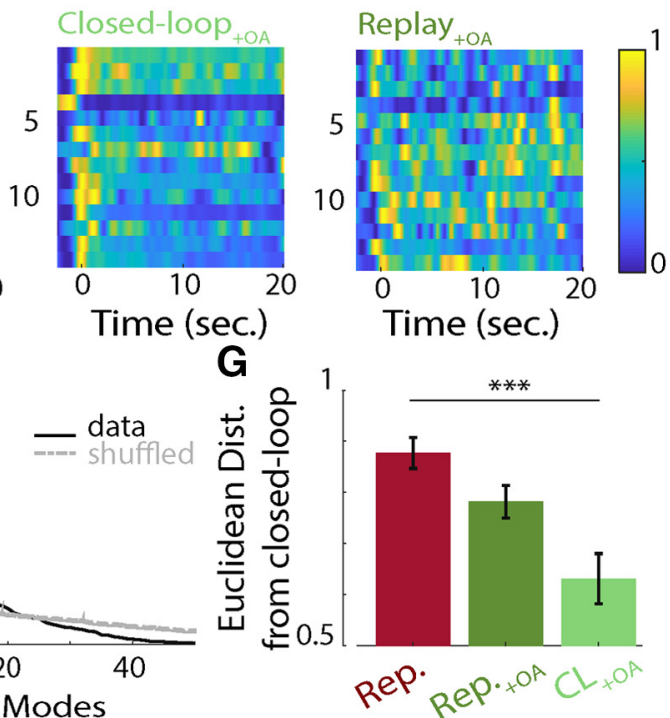

Figure 4. A, Forward velocity (in millimeters per second) during closed-loop trials (blue), replay trials (red), and closed-loop and replay trials with OA bath application (light green and dark green, respectively). The black lines represent the means. The average forward velocity is higher when $0 \mathrm{~A}$ is delivered (Kruskal-Wallis test, forward velocity: saline versus $0 \mathrm{~A}$, $\mathrm{df}=1$, $\chi^{2}=56.52, p<0.001$ ). $B$, As in $A$, except for the measured angular velocity (in degrees per second) for the different experimental treatments. OA triggered a tendency to turn right (Kruskal-Wallis test; angular velocity, saline vs $\mathrm{OA}: \mathrm{df}=1, \chi^{2}=75.17, p<0.001$ ). This tendency was more pronounced in replay than closed loop (Wilcoxon signed-rank test; angular velocity, closed loop $+O A$ vs replay $+O A: p<0.001$ ). C, Raster plot of the response of a unit to a visual stimulus in closed-loop trials (blue), replay trials (red), and closed-loop and replay trials with bath application of $O A$ (light green and dark green respectively). D, The normalized mean firing rates for 14 units during closed-loop trials, replay trials, and closed-loop and replay trials with bath application of $\mathrm{OA}$ (from left to right). $\boldsymbol{E}$, Projection of the closed-loop trials (blue), replay trials (red) and closed-loop and replay trials with OA bath application (light green and dark green, respectively) on modes 2 and 3 after SVD. The ellipses correspond to the $50 \%$ confidence interval. $\boldsymbol{F}$, Distribution of the singular values across mode for the firing rate responses (closedloop, replay without $O A$ and closed loop, and replay with $O A$ data) and its shuffled version (shuffled data). $\mathbf{G}$, Euclidean distance from the closed-loop trials to the replay trials with or without $O A$ application and the closed-loop trials with $\mathrm{OA}$ application. Application of $0 \mathrm{~A}$ and the behavioral control variable impacted the similarity between population responses to a visual stimulus (Kruskal-Wallis test: $\mathrm{df}=2, \chi^{2}=13.49, p=0.001$ ). Population responses in replay trials with bath application of OA were more similar to closed-loop than replay trials without $0 \mathrm{~A}$ (Wilcoxon signed rank test, $p=0.02)$. ***p $<0.001$. NS, not significant.

trials than the replay or unstimulated (ITI) trials (Fig. $3 H$ ). Classification by the algorithm was better than a random chance for both training and testing (Fig. 3I).

\section{OA application increased similarity between closed-loop and replay trials}

Neuroamines play key roles in regulating neural activity during different locomotion states, and OA is well known to be involved in the modulation of neural activity during insect flight (Maimon et al., 2010; Suver et al., 2012; Tuthill et al., 2014). To explore the potential participation of OA in the modulation we observed during closed-loop trials, we superfused the brain with $\mathrm{OA}\left(10^{-6} \mathrm{M}\right)$ while recording neural and behavioral activity. Both forward and angular velocities of honeybees increased after OA application (Kruskal-Wallis test for saline vs $\mathrm{OA}$; forward velocity: $\mathrm{df}=1, \chi^{2}=56.52$, $p<0.001$; angular velocity: $\chi^{2}=75.17, p<0.001$; Fig. $\left.4 A, B\right)$. Neural responses also reflected OA modulation, as follows. Before OA application, 6 of the 14 units recorded showed significant differences in firing rate between closed-loop and replay trials (Wilcoxon rank-sum test; $\Delta \mathrm{FR}$ vs $0, p<0.05$; $\Delta \mathrm{FR}$ vs bootstrapped $\Delta \mathrm{FR}, p<0.05)$. After OA application, firing rate responses did not differ significantly between the two stimulus conditions (Fig. 4D). In 12 of 14 units, OA evoked an increase in firing rate during both closed-loop and replay trials (Wilcoxon signed-rank test; baseline firing rate with OA vs saline, $p<0.05$; Fig. $4 C$ ). However, the application of OA during replay did not completely recapitulate the ensemble response dynamics observed in closed loop (Fig. 4D). Nonetheless, SVD applied to the ensemble firing rate responses showed separation between the groups in the first modes (Fig. $4 E$ ) and was reflected in the Euclidean distance in the first 20 modes (i.e., the modes with singular values above the noise model of all experimental trials; Fig. 4F), in which OA increased the similarity between replay and closed loop (Wilcoxon signed-rank test, $\mathrm{W}=88, p=0.02$; Fig. $4 G$ ).

Fixation behavior is dependent on the visual stimulus and is preceded by an increase in firing rate in medulla neurons The differences in neural responses between closed loop and replay motivated us to examine how the response dynamics change as the honeybee first begins to fixate on the visual stimulus. During closed-loop trials, 9 of 11 bees (82\%) fixated for 2-4 s (Fig. 5A). In 13 units, we observed an increase in firing rate preceding fixation (Fig. $5 B-E$; Wilcoxon signed-rank test, $p<0.05$ ). This increase could not be explained solely by the position of the 


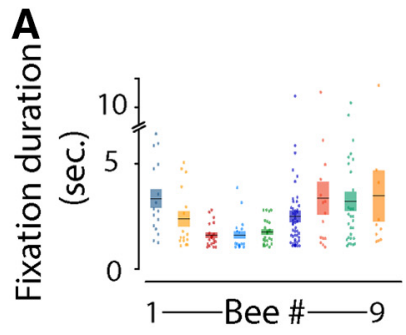

B

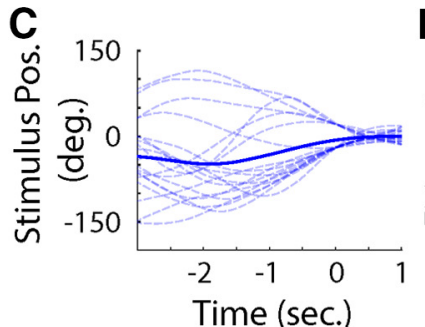

$\mathbf{F}$

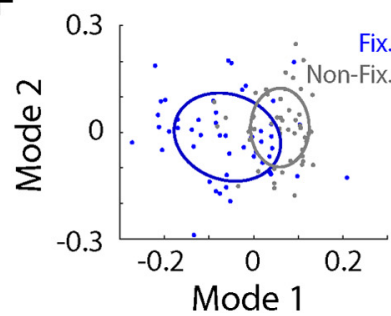

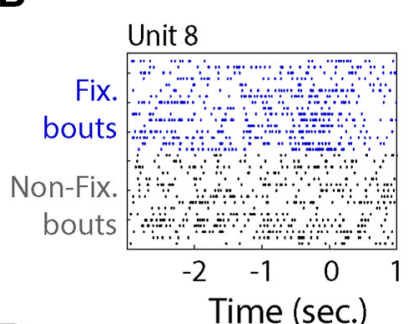

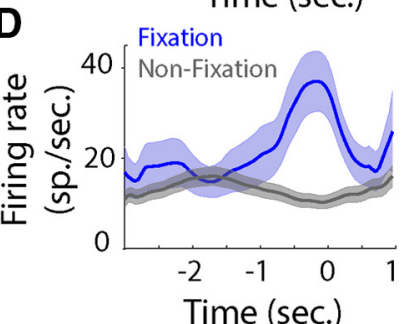

$G$

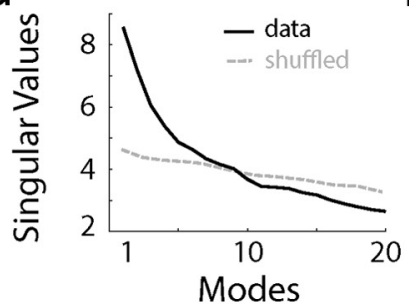

E
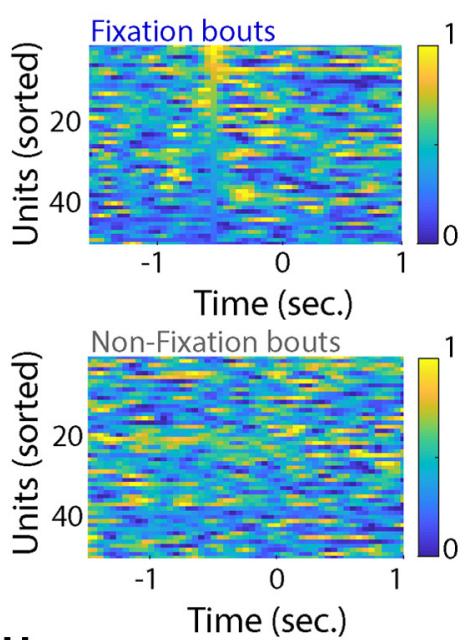

H

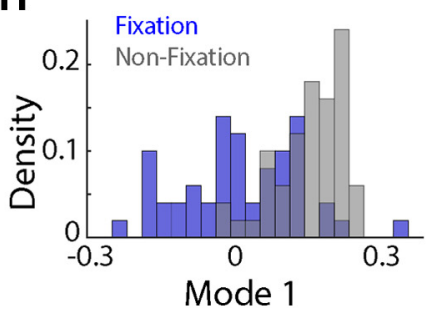

Figure 5. $\boldsymbol{A}$, Duration (in seconds) of fixation bouts for each bee. The mean $\pm \mathrm{SEM}$ is represented by a rectangle. $\boldsymbol{B}$, Raster plot of the response of a unit before ( $-3 \mathrm{~s}$ ) and during fixation (Fix.), or when the bee was not fixating (Non-Fix. bouts). Note the increase in firing rate just before fixation. $\boldsymbol{C}$, Position of the stimulus on the screen ( $\left.{ }^{\circ}\right) \mathrm{s}$ before to $1 \mathrm{~s}$ during fixation for the same unit as represented in $\boldsymbol{B}$. $\boldsymbol{D}$, Mean \pm SEM firing rate (in spikes per second) centered around fixation for the same unit as in $\boldsymbol{B}$ and $\boldsymbol{C}$. $\boldsymbol{E}$, The normalized mean firing rate for the neural ensemble during fixation (top) and randomly selected bouts (bottom). $\boldsymbol{F}$, Projection in the first two modes after SVD showed little separation between fixation bouts (blue dots) and randomly selected bouts (gray dots). The ellipses correspond to the $50 \%$ confidence interval. $\mathbf{G}$, Singular values across mode. The first 10 modes explain more of the variance than a shuffled and labelremoved version of the data. $\boldsymbol{H}$, Density distribution in the first mode for the fixation bouts (blue) and random samples (gray). Fixation (blue) and random (gray) values are transparent to show their overlap.

visual stimulus on the screen (Fig. 5B-D). To further explore the impact of fixation on those neurons, we extracted the spiking activity during the first second of all fixation bouts in the closedloop trials, as well as during the preceding second. Using these data, we built a "fixation-triggered average" of firing rate responses for all units in the ensemble, as well as the ensemble firing rate responses when the bees were not fixating on the stimulus (nonfixating). When the bee was not fixating, data were randomly selected during these periods. The resulting covariance matrices had higher eigenvalues in the first mode for the fixation matrix than the corresponding nonfixating one for all neurons exhibiting the prefixation increase in firing rate. To explore the impact of fixation on the population, we averaged the firing rate across fixation and nonfixating bouts for all units, including units that showed no difference between fixation and nonfixating bouts (Fig. 5E). After SVD analysis, the two populations separated significantly across the first mode (Kruskal-Wallis test, $V_{\text {closed-loop }}$ vs $V_{\text {replay, }} \mathrm{df}=1, \chi^{2}=33.69, p<0.001$; Fig. $\left.5 F-H\right)$.

\section{$\mathrm{OA}$ in the medulla is critical for fixation behavior}

To further explore how OA influences the sensitivity of visual responses and feedback during closed loop, we tested the ability of honeybees to fixate a visual stimulus at different levels of motor gain, and under different pharmacological interventions. Immediately before the experiment, either the OA receptor antagonist epinastine $\left(4 \times 10^{-3} \mathrm{M}\right)$ or saline control was focally microinjected into both medullas. For saline-injected bees, the duration of fixation bouts differed significantly among individual bees and with gain, with increased duration of fixation bouts at gains of -2 to 2 (two-way ANOVA; duration of fixation $\sim$ gain * bee ID: gain, $F=2.49, p=0.006$; bee ID: $F=3.32, p<0.001$; interaction factor: $F=1.22, p=0.146 ; n=36$; Fig. $6 A, B)$. Salineinjected bees significantly oriented toward and fixated on the visual stimulus; however, bees injected with epinastine were unable to maintain the visual stimulus in the frontal field of view (Watson's two-sample test of homogeneity; test statistic $=318.22$, $p<0.001$; Fig. 6C). Saline-injected bees showed the same pattern as that in uninjected bees, with a significant increase in duration of fixation bouts at gains of 0.1-1.0 (Kruskal-Wallis test; duration of fixation $\sim$ gain, $\chi^{2}=18.04, \mathrm{df}=4, p=0.001$; Fig. $\left.6 D\right)$. By contrast, epinastine-injected bees showed no increase in duration and fixated for a similar amount of time at all gains (KruskalWallis test; duration of fixation $\sim$ gain, $\chi^{2}=7.53, \mathrm{df}=4, p=0.11$; Fig. 6D). Overall, the injection of epinastine significantly decreased the duration of fixation bouts (Kruskal-Wallis test, duration of fixation $\sim$ injection type: $\chi^{2}=11.13, \mathrm{df}=1, p<$ 0.001; Fig. $6 D$ ). Similar to the results for the duration of fixation, the proportion of trials with fixation increased in saline-injected bees at gain levels from -5 to -1.5 , and decreased thereafter (Fig. 6E). In epinastine-injected bees, however, the proportion of trials with at least one instance of fixation did not change with gain level, and was lower than in saline-injected bees (Fig. 6E). In addition, epinastine-injected bees had higher forward velocities and lower angular velocities, and walked longer distances than saline-injected bees (Kruskal-Wallis test, distance walked injection type: $\chi^{2}=51.67, \mathrm{df}=1, p<0.001$; Fig. $\left.6 F, G\right)$. 

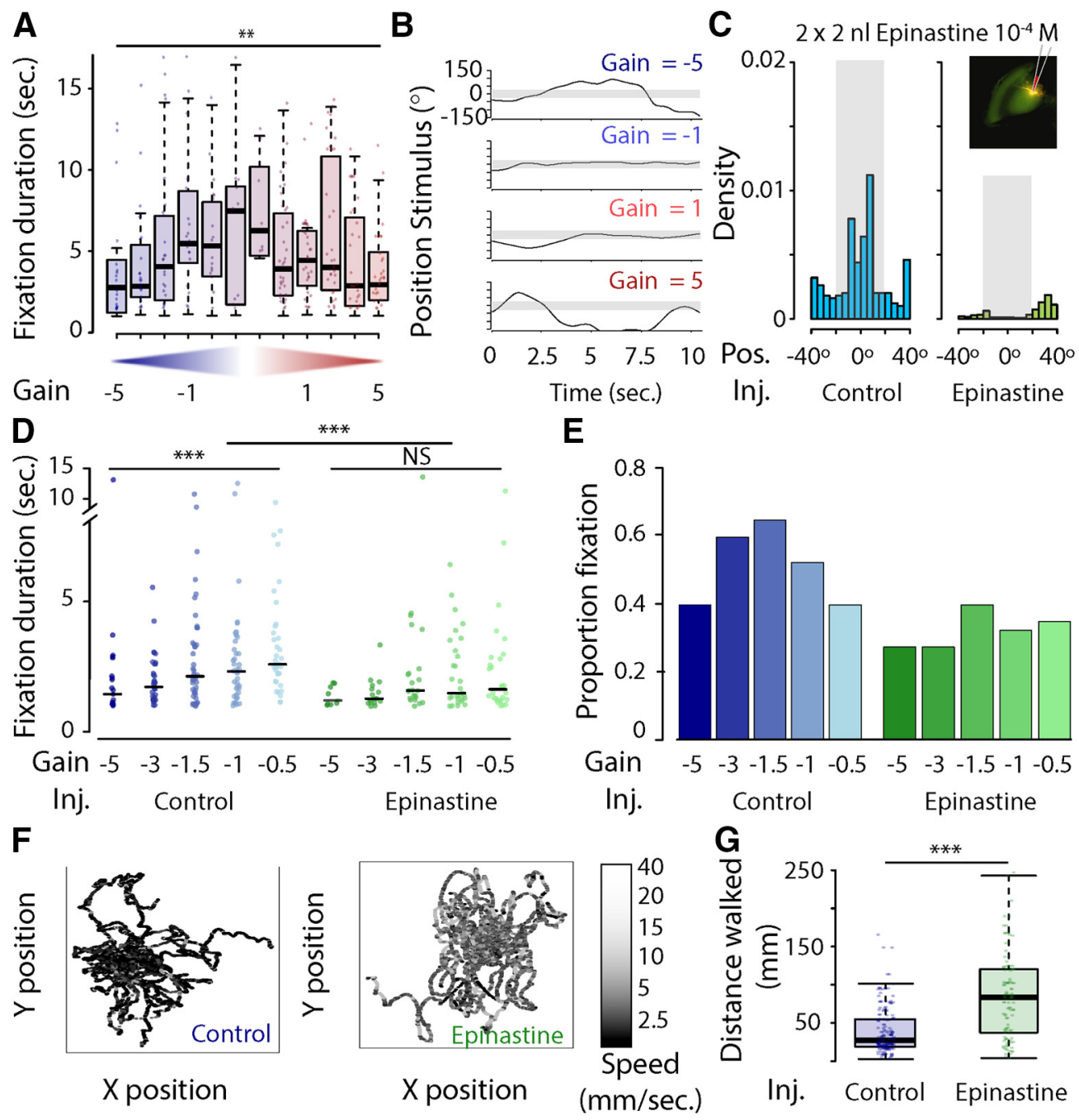

Figure 6. $A$, Duration (in seconds) of fixation across different levels of motor gain $(-5 ;-3 ;-1.5 ;-1 ;-0.5 ;-0.1 ; 0.1 ; 0.5 ; 1 ; 1.5 ; 3$; and 5$)$. Duration was significantly different across gain and honeybee (two-way ANOVA; duration of fixation bouts $\sim$ gain $*$ bee ID, gain: $F=2.49, p=0.006$; bee ID: $F=3.322, p<0.001$; interaction factor: $F=1.222, p=0.146, n=36$ ). $\boldsymbol{B}$, Position of the stimulus on the screen during the first $10 \mathrm{~s}$ of a trial for four different gains $(-5,-1,1,5)$. C, Distribution of bar position (in degrees) for all trials for a saline-injected bee (blue) and an epinastine-injected bee (green). The insert shows the injection in the medulla. $\boldsymbol{D}$, Duration of the fixation bouts (in seconds) across different levels of motor gain $(-5$; -3 ; -1.5 ; -1 ; and -0.5 ) in saline-injected (blue, $n=11$ ) and epinastine-injected bees (green, $n=10$ ). The duration of fixation was significantly different across levels of motor gain in salineinjected bees (Kruskal-Wallis test; duration of fixation $\sim$ gain: $\chi^{2}=18.048, \mathrm{df}=4, p=0.001$ ) but not for epinastine-injected bees (Kruskal-Wallis test: duration of fixation $\sim$ gain, $\chi^{2}=7.533, \mathrm{df}=4, p=0.11$ ). The overall duration of fixation was different between saline-injected and epinastine-injected bees (Kruskal-Wallis test, duration of fixation $\sim$ injection type: $\chi^{2}=11.132, \mathrm{df}=1, p<0.001$ ). $\boldsymbol{E}$, Proportion of trials with at least one fixation event and across levels of motor gain for saline-injected bees (blue) and epinastine-injected bees (green). $\boldsymbol{F}$, Fictive path of saline-injected (left) and epinastine-injected (right) bees at all levels of motor gain and colored according to their forward velocity (in millimeters per second). $\mathbf{G}$, Overall distance walked during trials for saline-injected (blue) and epinastine-injected (green) bees. Epinastine-injected bees walked larger distances than saline-injected bees (Kruskal-Wallis test; distance walked injection type: $\left.\chi^{2}=51.672, \mathrm{df}=1, p<0.001\right) . * * p<0.01 ; * * * p<0.001$. NS, not significant.

To ensure that the decreased fixation observed in epinastineinjected bees was not a result of higher walking velocity, we developed a random walk model and compared the duration of fixation bouts of fictive bees with the collected data. In the model, fictive bees could turn right, left, or go forward with the same probability and with an angular velocity drawn randomly from the distributions observed in saline-injected or epinastineinjected honeybees at a motor gain of -1 (Kruskal-Wallis test; instantaneous angular velocity $\sim$ injection type, $p<0.001$ ). As in actual bees, the modeled duration of fixation of fictive bees was shorter when based on data collected in epinastine-injected bees than in saline-injected bees (Kruskal-Wallis test; duration of fixation $\sim$ type of fictive bees, $p<0.001$ ). However, fictive bees based on the epinastine-injected data fixated on the visual stimulus in 55\% of trials, whereas fictive bees based on the saline-injected data fixated in only $47 \%$ of trials. This last result is the opposite of what we observed experimentally (Fig. 6E), demonstrating that a decrease of angular velocity associated with greater forward velocity cannot fully explain the impact of $\mathrm{OA}$ antagonist on fixation behavior.

\section{Discussion}

The brain is inherently a closed-loop system. The nervous system processes sensory signals from the environment to shape behavior, and behaviors consequently modify the environment. In the present study, we combined behavioral and multichannel recordings in walking honeybees to explore how behavioral state and sensorimotor feedback modulate visual processing in the honeybee medulla. Our results showed that when honeybees had behavioral control over the horizontal displacement of the visual scene, a subset of spiking neurons exhibited increased responses during the duration 
of the stimulus and before the onset of behavioral fixation, which was in contrast with results during their replay in open loop.

\section{Behavioral modulation of visual processing}

The behavioral context dependence of the neural response raises the question of mechanism. OA is an important neuromodulator of visual processing in many invertebrates, and in insects has been shown to play an important role during locomotion (Stern et al., 1995; Longden and Krapp, 2010; Jung et al., 2011; Suver et al., 2012; Tuthill et al., 2014; Strother et al., 2018). In honeybees, OA neurons innervate the three optic lobes with a relatively homogeneous distribution (Bicker, 1999), whereas only the medulla and the lobula are innervated in the fruit fly (Busch et al., 2009). In locusts, OA neurons with dense arborizations in the optic lobes were proposed to participate in mediating dishabituation or arousal of the visual system (Stern et al., 1995). In our honeybee study, the application of OA triggered a general increase in gain in the neural population, similar to that observed during closed-loop stimulation, in which the honeybee had behavioral control over the visual scene (Fig. $4 C, G$ ). The speed tuning of visual processing was consistent with efficient coding, wherein the tuning of neurons should match that of the environmentwhen shifting from nonactive behavioral bouts to walking or flying bouts, the optic flow shifts toward higher frequencies.

Our study adds to the ongoing redefinition of insect visual processing as more than a strictly feedforward processing stream from the lamina to the lobula. Flying insects live in a fast world: a bumblebee, for example, flies at $7.1 \mathrm{~m} / \mathrm{s}$, or $\sim 400$ body lengths $/ \mathrm{s}$ (Riley et al., 1999). Behavioral modulation of visual processing may ensure correct and rapid behavioral responses under different environmental conditions-as image frequencies increase during flight, or decrease during hovering-while reducing neuronal energy consumption by dynamically altering neuron sensitivity according to locomotion state (Niven, 2016). To our knowledge, optic lobe neuronal recordings have not been performed in nonactive, walking, and flying locomotion states in the same individual. Such recordings would facilitate better understanding of how neuronal activity is modulated across the different locomotion states. Moreover, different species must rely on different strategies to process visual stimuli. For instance, the extensively studied lobula plate of the fruit fly is absent in the honeybee. Comparative studies are thus critical to achieve a more thorough understanding of neural modulation.

\section{Adaptive control of visual processing}

Using virtual reality, researchers have shown that insects can modify their motor patterns to achieve a goal. Fruit flies placed in a flying arena can modify their leg motion to achieve steering at both positive and negative levels of motor gain (i.e., the relationship between the insect motion and visual scene motion; Wolf et al., 1992). Such behavior demonstrates a high level of motor pattern flexibility, and the need to combine visual processing with information from locomotion. In our experiment, honeybees showed strong aptitude for adapting their locomotion to orient toward a visual stimulus (Fig. 6A). We then asked whether OA was important for adapting motion to obtain the expected visual motion. Microinjection of an OA antagonist into the medulla disrupted fixation behavior, with loss of adaptive responses to different levels of motor gain, suggesting that $\mathrm{OA}$ is involved in visual response tuning (Fig. $6 D, E$ ).

\section{Behavioral control and visual learning}

Operant behavior has classically been used in learning protocols. The ability to learn the consequence of a particular action is critical for adaptive behavior and thus for survival. Previous studies have shown that the presence of optic flow is critical for visual learning in tethered honeybees (Buatois et al., 2017; Rusch et al., 2017), and it is possible that behavioral control will prove critical for complex learning protocols, such as the learning of abstract concepts. Over the course of our closed-loop trials, we did not identify changes in behavior or neural activity that would indicate that the bees are learning. Although our experiment did not explicitly use an operant conditioning paradigm - such as using positive reinforcement, like nectar, paired with the closedloop visual stimulus-future studies could easily incorporate this aspect into the experimental design. One means of ensuring extraction of only the most relevant signals during learning, while dedicating the least possible amount of computational resources, can be categorized when an animal attends to a visual object (Logan, 1992). In the presence of competing stimuli, selective fixation allows an animal to respond to specific visual objects, permitting some stimuli to evoke a behavioral response while ignoring others. Visual fixation has been studied extensively in primates (for review, see Wolfe, 2000). For instance, attention alters the synchrony of a neuronal population (Fries et al., 2001) and can modulate both the firing rate and the receptive field of neurons (for review, see McAdams and Maunsell, 1999; Treue, 2001). An overall boost in signal gain, comparable to the increase in firing rate we observed in honeybees, has been described in the visual cortex of mice (for review, see Niell and Stryker, 2010; Schneider, 2020). Recent studies in fruit flies have shown that visual salience increases neural activity at a specific frequency, and may be correlated to behavioral selection and suppression (van Swinderen and Greenspan, 2003; van Swinderen, 2007, 2012; Tang and Juusola, 2010). However, these studies relied on measures of local field potential, which is proposed to reflect the synchronous activity of a population of neurons. While we did not present competing stimuli in our study, we did observe an increase in firing rate that preceded fixation behavior during closed-loop trials (Fig. $5 E$ ). This finding is consistent with the local field potential change in the medulla that was observed to precede fixation on one of two competing stimuli (Paulk et al., 2014). Behavioral experiments that record activity in multiple areas of the brain while presenting the animal with distractors will shed more light on attention-like processes, and their role during learning.

Our results emphasize the importance of behavioral closedloop control on visual processing in the medulla, and its interplay with behavioral and internal-state modulation. The medulla has received less attention than the lobula in studies of behavioral and internal-state modulation, although a single fruit fly medulla column contains $\sim 900$ neurons, $>2500$ presynaptic sites, and $>300,000$ postsynaptic sites (Takemura et al., 2015). The precise location in the visual pathway that integrates feedback from leg or wing position is still unknown. Answering this question will likely require simultaneous recordings in different brain areas, and better knowledge of the connectomics of the visual pathway. Given the impressive amount of processing taking place in the medulla, it may be a strong candidate for behavioral and internal-state modulation during visual processing.

\section{References}

Alem S, Perry CJ, Zhu X, Loukola OJ, Ingraham T, Søvik E, Chittka L (2016) Associative mechanisms allow for social learning and cultural transmission of string pulling in an insect. PLoS Biol 14:e1002564.

Bahl A, Ammer G, Schilling T, Borst A (2013) Object tracking in motionblind flies. Nat Neurosci 16:730-738.

Bicker G (1999) Biogenic amines in the brain of the honeybee: cellular distribution, development, and behavioral functions. Microsc Res Tech 44:166-178. 
Brembs B (2009) The importance of being active. J Neurogenet 23:120-126.

Buatois A, Pichot C, Schultheiss P, Sandoz JC, Lazzari CR, Chittka L, Avarguès-Weber A, Giurfa M (2017) Associative visual learning by tethered bees in a controlled visual environment. Sci Rep 7:12903.

Busch S, Selcho M, Ito K, Tanimoto H (2009) A map of octopaminergic neurons in the Drosophila brain. J Comp Neurol 513:643-667.

Chiappe ME, Seelig JD, Reiser MB, Jayaraman V (2010) Walking modulates speed sensitivity in Drosophila motion vision. Curr Biol 20:1470-1475.

De Bivort BL, Van Swinderen B (2016) Evidence for selective attention in the insect brain. Curr Opin Insect Sci 15:9-15.

Dyer AG, Neumeyer C, Chittka L (2005) Honeybee (Apis mellifera) vision can discriminate between and recognise images of human faces. J Exp Biol 208:4709-4714

Fenk LM, Poehlmann A, Straw AD (2014) Asymmetric processing of visual motion for simultaneous object and background responses. Curr Biol 24:2913-2919.

Fries P, Reynolds JH, Rorie AE, Desimone R (2001) Modulation of oscillatory neuronal synchronization by selective visual attention. Science 291:15601563.

Giurfa M, Vorobyev M (1997) The detection and recognition of color stimuli by honeybees: performance and mechanisms. Israel J Plant Sci 45:129-140.

Giurfa M, Zhang S, Jenett A, Menzel R, Srinivasan MV (2001) The concepts of "sameness" and "difference" in an insect. Nature 410:930-933.

Green J, Adachi A, Shah KK, Hirokawa JD, Magani PS, Maimon G (2017) A neural circuit architecture for angular integration in Drosophila. Nature 546:101-106.

Heisenberg M, Wolf R, Brembs B (2001) Flexibility in a single behavioral variable of Drosophila. Learn Mem 8:1-10.

Hori S, Takeuchi H, Arikawa K, Kinoshita M, Ichikawa N, Sasaki M, Kubo T (2006) Associative visual learning, color discrimination, and chromatic adaptation in the harnessed honeybee Apis mellifera L. J Comp Physiol A Neuroethol Sens Neural Behav Physiol 192:691-700.

Hori S, Takeuchi H, Kubo T (2007) Associative learning and discrimination of motion cues in the harnessed honeybee Apis mellifera L. J Comp Physiol A Neuroethol Sens Neural Behav Physiol 193:825-833.

Jung SN, Borst A, Haag J (2011) Flight activity alters velocity tuning of fly motion-sensitive neurons. J Neurosci 31:9231-9237.

Khan AG, Hofer SB (2018) Contextual signals in visual cortex. Curr Opin Neurobiol 52:131-138.

Koay SA, Thiberge SY, Brody C, Tank DW (2019) Neural correlates of cognition in primary visual versus neighboring posterior cortices during visual evidence-accumulation-based navigation. bioRxiv. doi: https://doi.org/ $10.1101 / 568766$

Logan GD (1992) Attention and preattention in theories of automaticity. Am J Psychol 105:317-339.

Longden KD, Krapp HG (2010) Octopaminergic modulation of temporal frequency coding in an identified optic flow-processing interneuron. Front Syst Neurosci 4:153.

Loukola OJ, Perry CJ, Coscos L, Chittka L (2017) Bumblebees show cognitive flexibility by improving on an observed complex behavior. Science 355:833-836.

Maimon G, Straw AD, Dickinson MH (2010) Active flight increases the gain of visual motion processing in Drosophila. Nat Neurosci 13:393-399.

McAdams CJ, Maunsell JH (1999) Effects of attention on orientation-tuning functions of single neurons in macaque cortical area V4. J Neurosci 19:431-441.

Moore RJ, Taylor GJ, Paulk AC, Pearson T, van Swinderen B, Srinivasan MV (2014) FicTrac: a visual method for tracking spherical motion and generating fictive animal paths. J Neurosci Methods 225:106-119.

Mota T, Giurfa M, Sandoz JC (2011) Color modulates olfactory learning in honeybees by an occasion-setting mechanism. Learn Mem 18:144-155.

Niell CM, Stryker MP (2010) Modulation of visual responses by behavioral state in mouse visual cortex. Neuron 65:472-479.

Niggebrügge C, Leboulle G, Menzel R, Komischke B, de Ibarra NH (2009) Fast learning but coarse discrimination of colours in restrained honeybees. J Exp Biol 212:1344-1350.

Niven JE (2016) Neuronal energy consumption: biophysics, efficiency and evolution. Curr Opin Neurobiol 41:129-135.

Nobre AC, Kastner S (2014) Handbook of attention. Oxford: OUP.
Paulk AC, Stacey JA, Pearson TW, Taylor GJ, Moore RJ, Srinivasan MV, Van Swinderen B (2014) Selective attention in the honeybee optic lobes precedes behavioral choices. Proc Natl Acad Sci U S A 111:5006-5011.

Paulk AC, Kirszenblat L, Zhou Y, van Swinderen B (2015) Closed-loop behavioral control increases coherence in the fly brain. J Neurosci 35:10304-10315.

R Core Team (2017) R: a language and environment for statistical computing. Vienna, Austria: R Foundation for Statistical Computing.

Rein J, Mustard JA, Strauch M, Smith BH, Galizia CG (2013) Octopamine modulates activity of neural networks in the honey bee antennal lobe. J Comp Physiol A Neuroethol Sens Neural Behav Physiol 199:947-962.

Reiser MB, Dickinson MH (2008) A modular display system for insect behavioral neuroscience. J Neurosci Methods 167:127-139.

Riley JR, Reynolds DR, Smith AD, Edwards AS, Osborne JL, Williams IH, McCartney HA (1999) Compensation for wind drift by bumble-bees. Nature 400:126-126.

Roeder T (1999) Octopamine in invertebrates. Prog Neurobiol 59:533-561.

Roeder T, Degen J, Gewecke M (1998) Epinastine, a highly specific antagonist of insect neuronal octopamine receptors. Eur J Pharmacol 349:171-177.

Rusch C, Roth E, Vinauger C, Riffell JA (2017) Honeybees in a virtual reality environment learn unique combinations of colour and shape. J Exp Biol 220:3478-3487.

Sakura M, Okada R, Aonuma H (2012) Evidence for instantaneous e-vector detection in the honeybee using an associative learning paradigm. Proc Biol Sci 279:535-542.

Schneider DM (2020) Reflections of action in sensory cortex. Curr Opin Neurobiol 64:53-59.

Stern M, Thompson KSJ, Zhou P, Watson DG, Midgley JM, Gewecke M, Bacon JP (1995) Octopaminergic neurons in the locust brain: morphological, biochemical and electrophysiological characterisation of potential modulators of the visual system. J Comp Physiol A Neuroethol Sens Neural Behav Physiol 177:611-625.

Strother JA, Wu ST, Wong AM, Nern A, Rogers EM, Le JQ, Rubin GM, Reiser MB (2017) The emergence of directional selectivity in the visual motion pathway of Drosophila. Neuron 94:168-182.

Strother JA, Wu ST, Rogers EM, Eliason JL, Wong AM, Nern A, Reiser MB (2018) Behavioral state modulates the ON visual motion pathway of Drosophila. Proc Natl Acad Sci U S A 115:E102-E111.

Suver MP, Mamiya A, Dickinson MH (2012) Octopamine neurons mediate flight-induced modulation of visual processing in Drosophila. Curr Biol 22:2294-2302.

Takemura S-y, Xu CS, Lu Z, Rivlin PK, Parag T, Olbris DJ, Plaza S, Zhao T, Katz WT, Umayam L, Weaver C, Hess HF, Horne JA, Nunez-Iglesias J, Aniceto R, Chang L-A, Lauchie S, Nasca A, Ogundeyi O, Sigmund C, et al (2015) Synaptic circuits and their variations within different columns in the visual system of Drosophila. Proc Natl Acad Sci U S A 112:13711-13716.

Tang S, Juusola M (2010) Intrinsic activity in the fly brain gates visual information during behavioral choices. Nat Proc 5:e14455.

Tedjakumala SR, Aimable M, Giurfa M (2014) Pharmacological modulation of aversive responsiveness in honey bees. Front Behav Neurosci 7:221.

Treue S (2001) Neural correlates of attention in primate visual cortex. Trends Neurosci 24:295-300.

Tuthill JC, Nern A, Rubin GM, Reiser MB (2014) Wide-field feedback neurons dynamically tune early visual processing. Neuron 82:887-895.

van Swinderen B (2007) Attention-like processes in Drosophila require short-term memory genes. Science 315:1590-1593.

van Swinderen B (2012) Competing visual flicker reveals attention-like rivalry in the fly brain. Front Integr Neurosci 6:96.

van Swinderen B, Greenspan RJ (2003) Salience modulates $20-30 \mathrm{~Hz}$ brain activity in Drosophila. Nat Neurosci 6:579-586.

Wolf R, Heisenberg M (1986) Visual orientation in motion-blind flies is an operant behaviour. Nature 323:154-156.

Wolf R, Heisenberg M (1991) Basic organization of operant behavior as revealed in Drosophila flight orientation. J Comp Physiol A Neuroethol Sens Neural Behav Physiol 169:699-705.

Wolf R, Voss A, Hein S, Heisenberg M (1992) Can a fly ride a bicycle? Philos Trans R Soc Lond B Biol Sci 337:261-269.

Wolfe JM (2000) Visual attention. In: Seeing (De Valois KK ed), pp 335-386. San Diego: Academic. 